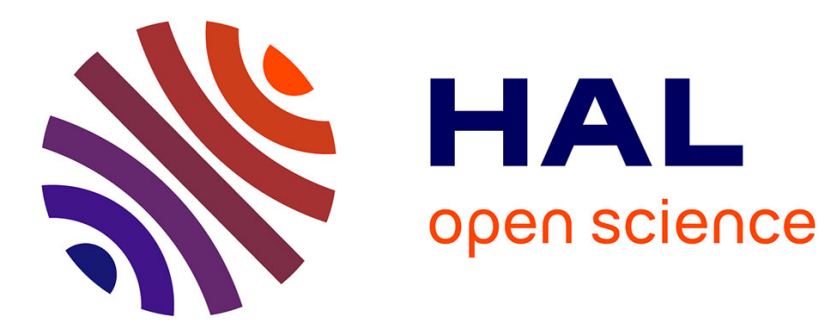

\title{
Iron-catalyzed benzamide formation. Application to the synthesis of moclobemide.
}

Xavier Bantreil, Nasreddine Kanfar, Nicolas Gehin, Ethan Golliard, Pauline Ohlmann, Jean Martinez, Frédéric Lamaty

\section{- To cite this version:}

Xavier Bantreil, Nasreddine Kanfar, Nicolas Gehin, Ethan Golliard, Pauline Ohlmann, et al.. Ironcatalyzed benzamide formation. Application to the synthesis of moclobemide.. Tetrahedron, 2014, 70 (34), pp.5093-5099. 10.1016/j.tet.2014.06.001 . hal-01015316

\section{HAL Id: hal-01015316 https://hal.science/hal-01015316}

Submitted on 3 Mar 2021

HAL is a multi-disciplinary open access archive for the deposit and dissemination of scientific research documents, whether they are published or not. The documents may come from teaching and research institutions in France or abroad, or from public or private research centers.
L'archive ouverte pluridisciplinaire HAL, est destinée au dépôt et à la diffusion de documents scientifiques de niveau recherche, publiés ou non, émanant des établissements d'enseignement et de recherche français ou étrangers, des laboratoires publics ou privés. 


\title{
Iron-catalyzed benzamide formation. Application to the synthesis of moclobemide
}

\author{
Xavier Bantreil*, Nasreddine Kanfar, Nicolas Gehin, Ethan Golliard, Pauline Ohlmann, \\ Jean Martinez, Frédéric Lamaty* \\ Institut des Biomolécules Max Mousseron, UMR 5247 CNRS-UM I-UM II-ENSCM, Université Montpellier II, Place E. Bataillon, 34095 Montpellier Cedex \\ 5, France
}

Keywords:

Iron

Homogeneous catalysis

Amides

Oxidation

Green chemistry

\begin{abstract}
A B S T R A C T
A convenient and user-friendly method to yield benzamides from primary and secondary amines and various benzylic alcohols in the presence of a cheap iron salt $\left(\mathrm{FeCl}_{2} \cdot 4 \mathrm{H}_{2} \mathrm{O}\right)$ and tert-butylhydroperoxide (70\% in water) as a stoichiometric oxidant is described. Control experiments indicated that this reaction might involve radical species. This method proved to be general, generating a family of 30 benzamides and was applied to the preparative synthesis of anti-anxiety drug moclobemide.
\end{abstract}

\section{Introduction}

Amides are highly important functional groups due to their preponderance in many natural products such as peptides and proteins, pharmaceutical agents or man-made polymers, such as Nylon 6,6. ${ }^{1}$ In most cases, common techniques to generate an amide bond involve the use of toxic coupling agents and toxic solvents. On industrial scale, these drawbacks directly impact environment and economics. To circumvent such issues, organometallic methodologies to form amides directly from alcohols and amines have recently emerged. ${ }^{2}$ The general mechanism for this type of reaction involves the oxidation of an alcohol to the corresponding aldehyde, which will react with the amine to form a hemiaminal intermediate that is converted to the amide upon oxidation. It is important to note that in a competitive pathway, the aldehyde could be oxidized to the carboxylic acid, an undesired side-product (Scheme 1).

Efficient approaches have been reported using transition metals such as ruthenium ${ }^{3}$ and rhodium, ${ }^{4}$ and heterogeneous catalysts, ${ }^{5}$ such as $\mathrm{Au} / \mathrm{TiO}_{2}$ systems, ${ }^{6} \mathrm{Au} / \mathrm{DNA}$ nanohybrids, ${ }^{7}$ and aluminasupported silver clusters. ${ }^{8}$ Such approaches are highly attractive

\footnotetext{
* Corresponding authors. Tel.: +33 (0) 467143967; fax: +33 (0) 467144866; e-mail addresses: xavier.bantreil@univ-montp2.fr (X. Bantreil), frederic.lamaty@ univ-mont2.fr (F. Lamaty).
}

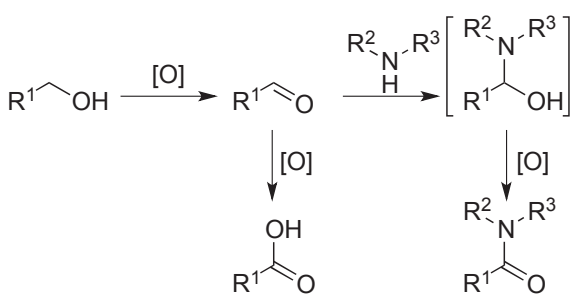

Scheme 1. General mechanism for amide formation.

as catalysis, step and atom economy are part of the 12 principles of green chemistry developed by Anastas and Warner. ${ }^{9}$

To compete with those techniques involving expensive metals, studies on copper- or iron-catalyzed formation of amides from aldehydes have been reported. ${ }^{10}$ As a part of our general interest in amide bonds, ${ }^{11}$ we recently reported the copper-catalyzed amidation directly from benzylic alcohols and amines, which allowed to go one-step further and realize a one-pot double oxidation. ${ }^{12}$ Similar approach using zinc was developed (Scheme 2 , left). ${ }^{13}$ In 2013, Porcheddu et al. reported the iron-catalyzed amidation of $\mathrm{N}$ chloramines, generated from toxic and non atom-economic $\mathrm{N}$ chlorosuccinimide, which required the use of 5 equiv of alcohols to obtain good yields (Scheme 2, top) ${ }^{14}$ More recently, Ghosh et al. reported a stepwise iron-catalyzed synthesis of benzamides, requiring a first step of oxidation of alcohol into aldehyde prior to 


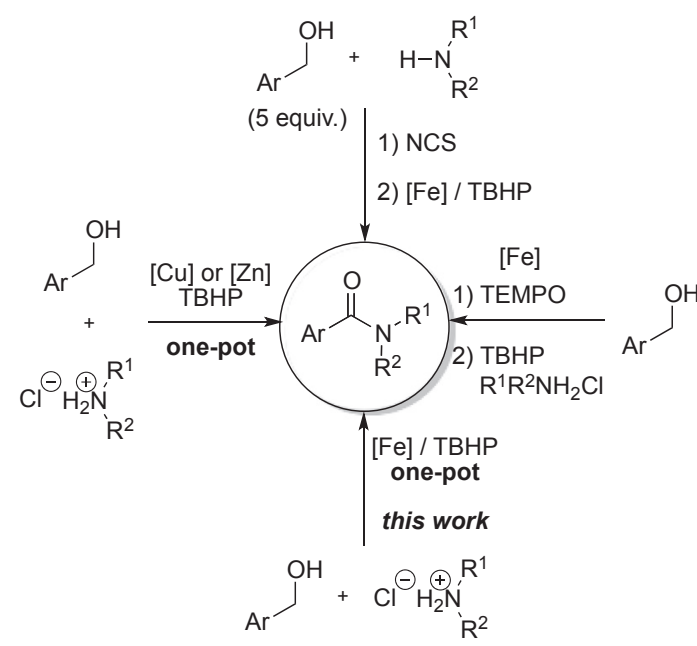

Scheme 2. Benzamide formation from alcohols in the litterature.

addition of the amine and TBHP (tert-butylhydroperoxide) as final oxidant (Scheme 2, right) ${ }^{15}$ In here, we want to report a one-pot two-steps convenient and practical method using a cheap iron salt as catalyst and readily available hydrochloric salt of amines $\left(\mathrm{TON}<8.9, \mathrm{TOF}<2.2 \mathrm{~h}^{-1}\right)$.

\section{Results/discussion}

Optimization of the reaction conditions was started using benzylic alcohol and cyclohexamine hydrochloric salt (1.5 equiv) in the presence of TBHP $\left(70 \%\right.$ in $\left.\mathrm{H}_{2} \mathrm{O}\right)$ as oxidant and an iron salt in refluxing acetonitrile. It is worth noting that initial attempts to perform the amidation directly from free amines failed, proving that masking of the nitrogen as its ammonium salt was compulsory to the reaction. As already stated for copper catalysis, ${ }^{12}$ calcium carbonate gave the best results. Its weak basicity and poor solubility induce a slow and controlled release of free amine within the reaction media. In the meantime, the alcohol would be oxidized to aldehyde that could react with free amine to yield corresponding amide after a second oxidation. This choice of base proved to be the key point for the feasibility of a one-pot two-steps reaction.

Screening of iron salts was realized. Apart from $\mathrm{K}_{4} \mathrm{Fe}(\mathrm{CN})_{6} \cdot 3 \mathrm{H}_{2} \mathrm{O}$ that gave only $53 \%$ conversion, all sources of iron tested gave around $80-85 \%$ conversion independently of the oxidation state of the metal (Table 1 , entries $1-6$ ). The best results were obtained using $\mathrm{FeCl}_{2} \cdot 4 \mathrm{H}_{2} \mathrm{O}$ and $\mathrm{FeCl}_{3}$, giving $87 \%$ conversion of amide 3aa. As $\mathrm{FeCl}_{2} \cdot 4 \mathrm{H}_{2} \mathrm{O}$ is less corrosive than $\mathrm{FeCl}_{3}$, the optimization was continued with this salt. Replacement of TBHP $\left(70 \%\right.$ in $\left.\mathrm{H}_{2} \mathrm{O}\right)$ by TBHP (5.5 $\mathrm{M}$ in nonane) or hydrogen peroxide induced a decrease to, respectively, $45 \%$ and $5 \%$ conversion (entries $7-8$ ).

As explained above (Scheme 1), the main side-product formed during the amidation is carboxylic acid. During our previous study on copper-catalyzed amidation, we realized that alcohols featuring electron-donating groups were prone to convert quickly to corresponding benzoic acids. Thus, in view to develop the most general conditions and increase the isolated yield of this reaction, a reverse of the amine/alcohol stoichiometry was envisioned (Table 2). While the use of 1.3 equiv of alcohols in the presence of 5 equiv of TBHP and $5 \mathrm{~mol} \%$ of $\mathrm{FeCl}_{2} \cdot 4 \mathrm{H}_{2} \mathrm{O}$ gave a $72 \%$ yield (entry 1 ), increasing the quantity of iron salt to $10 \mathrm{~mol} \%$ induced a satisfactory $89 \%$ yield (entry 2). Decreasing the excess of TBHP to 4 equiv did not affect the isolated yield ( $89 \%$, entry 3 ). As commercially available iron salts are often contaminated with other metals, the reaction was performed using extra pure iron source and base. Resulting amide was isolated in $82 \%$ (entry 4), indicating that traces of metals were not
Table 1

Optimization of reaction conditions ${ }^{\mathrm{a}}$

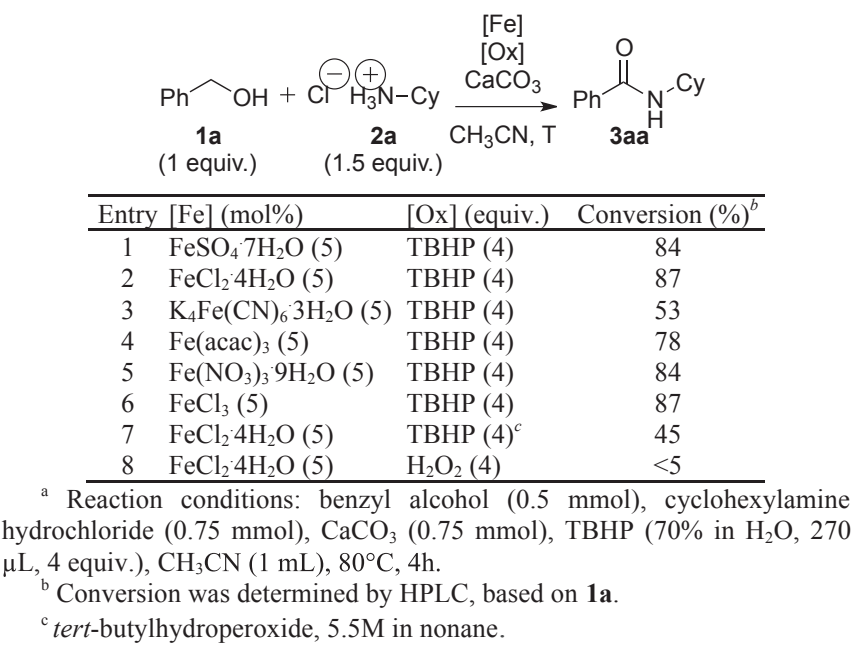

Table 2

Optimization of the reaction conditions with a reverse stoichiometry

\begin{tabular}{lllll}
\hline Entry & {$[\mathrm{Fe}](\mathrm{mol} \%)$} & {$[\mathrm{Ox}]($ equiv) } & Ratio 2a/1a & Yield $(\%)^{\mathrm{b}}$ \\
\hline 1 & $\mathrm{FeCl}_{2} \cdot 4 \mathrm{H}_{2} \mathrm{O}(5)$ & TBHP (5) & $1: 1.3$ & 72 \\
2 & $\mathrm{FeCl}_{2} \cdot 4 \mathrm{H}_{2} \mathrm{O}(10)$ & TBHP (5) & $1: 1.3$ & 89 \\
3 & $\mathrm{FeCl}_{2} \cdot 4 \mathrm{H}_{2} \mathrm{O}(10)$ & TBHP (4) & $1: 1.3$ & 89 \\
4 & $\mathrm{FeCl}_{2} \cdot 4 \mathrm{H}_{2} \mathrm{O}(10)^{\mathrm{C}}$ & TBHP (4) & $1: 1.3$ & 82 \\
5 & - & TBHP (4) & $1: 1.3$ & 27 \\
\hline
\end{tabular}

${ }^{\text {a }}$ Reaction conditions: Benzyl alcohol $(0.65 \mathrm{mmol})$, cyclohexylamine hydrochloride (0.5 mmol), $\mathrm{CaCO}_{3}(0.5 \mathrm{mmol}), \mathrm{TBHP}$ ( $70 \%$ in $\mathrm{H}_{2} \mathrm{O}, 270 \mu \mathrm{L}, 4$ equiv), $\mathrm{CH}_{3} \mathrm{CN}(1 \mathrm{~mL})$, $80{ }^{\circ} \mathrm{C}, 4 \mathrm{~h}$.

b Isolated yield, based on $\mathbf{2 a}$.

c $\mathrm{FeCl}_{2} \cdot 4 \mathrm{H}_{2} \mathrm{O} 99.99 \%$ and $\mathrm{CaCO}_{3} 99.995 \%$ pure were used.

responsible for the amidation reaction. Finally, a control experiment in the absence of iron salt indicated that the transition metal was necessary to the reaction ( $27 \%$ without $\mathrm{FeCl}_{2} \cdot 4 \mathrm{H}_{2} \mathrm{O}$, entry 5 ).

The scope of the reaction was then examined. First, variation of the amine was investigated (Table 3). For convenience, even if not specified, the amine hydrochloride salts were used throughout this study. Methyl and butyl amine reacted efficiently yielding 3ab and 3ac in 70\% and 76\%, respectively. Increasing the bulkiness with isopropyl and cyclohexylamine did not affect the amidation reaction (3ad, 72\%; 3aa, 89\%). Even bulkier tert-butyl amine gave 3ae in 59\% yield. Benzyl amine, which was shown to yield easily benzamide in the presence of iron and TBHP, ${ }^{16}$ was found to produce amide 3 af in $63 \%$ yield. Substituted benzylamines $2 \mathrm{~g}-\mathbf{i}$ furnished corresponding amides in yields spanning from 52\% to 86\%. Phenethyl and $p$ methoxyphenethyl amines $\mathbf{2} \mathbf{j}$ and $\mathbf{2 k}$ gave corresponding amides in moderate yields ( $\approx 60 \%$ ). Phenylalanine tert-butyl ester gave $77 \%$ of corresponding benzoylated aminoacid 3al. To our delight, the use of unprotected phenylalanine hydrochloric salt allowed the coupling in $70 \%$ yield (3am), indicating the tolerance of the conditions toward the carboxylic acid functionality. To the best of our knowledge, such reaction with substrate featuring a free acid had never been accomplished in previous reports. The iron-catalyzed amidation was also performed in the presence of more nucleophilic but also more hindered secondary amines. Although bearing two benzylic oxidable positions, dibenzylamine gave a good $81 \%$ yield of 3an. Cyclic amines reacted correctly, $\mathrm{HCl}-\mathrm{Pro}-{ }^{t} \mathrm{Bu}(\mathbf{2 o})$, piperidine (2p) and morpholine (2q) yielding the corresponding amides in, respectively, 55\% (3ao), 65\% (3ap) and 64\% (3aq).

The diversification was continued using various benzylic alcohols in the presence of $(\alpha)$-methylbenzyl amine $\mathbf{2 g}$ (Table 4). 
Table 3

Variation of primary and secondary amines in the amidation conditions

$$
\text { (1.3 equiv.) }
$$
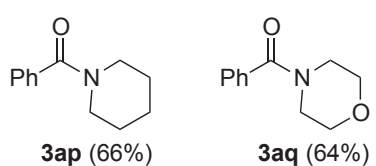

Reaction conditions: benzyl alcohol $(0.65 \mathrm{mmol})$, amine hydrochloride $\mathbf{2 a}$ q $(0.5 \mathrm{mmol}), \mathrm{FeCl}_{2} 4 \mathrm{H}_{2} \mathrm{O}(0.05 \mathrm{mmol}), \mathrm{CaCO}_{3}(0.5 \mathrm{mmol}), \mathrm{TBHP}(70 \%$ in $\mathrm{H}_{2} \mathrm{O}, 270 \mu \mathrm{L}, 4$ equiv. $), \mathrm{CH}_{3} \mathrm{CN}(1 \mathrm{~mL}), 80^{\circ} \mathrm{C}, 4 \mathrm{~h}$.

Table 4

Variation of benzylic alcohols in the amidation conditions

$$
\begin{aligned}
& \text { 1b-m } \\
& \stackrel{\mathrm{CaCO}_{3} \text { (1 equiv.) }}{\mathrm{CH}_{3} \mathrm{CN}, 80^{\circ} \mathrm{C}, 4 \mathrm{~h}} \text { 3bg-mg }
\end{aligned}
$$

Reaction conditions: benzyl alcohol $\mathbf{1 b}-\mathbf{m}(0.65 \mathrm{mmol})$, amine hydrochloride $\mathbf{2 g}(0.5$ $\mathrm{mmol}), \mathrm{FeCl}_{2} 4 \mathrm{H}_{2} \mathrm{O}(0.05 \mathrm{mmol}), \mathrm{CaCO}_{3}(0.5 \mathrm{mmol})$, T-Hydro $(270 \mu \mathrm{L}, 4$ equiv. $)$, $\mathrm{CH}_{3} \mathrm{CN}(1 \mathrm{~mL}), 80^{\circ} \mathrm{C}, 4 \mathrm{~h} .{ }^{a} 2$ equiv. of $p$-methoxybenzylalcohol were used.

Electron-donating substituents in para position gave a slight decrease in yield with $58 \%$ and $69 \%$ for, respectively, $3 \mathbf{b g}$ and $3 \mathbf{c g} . p$ Chlorobenzyl alcohol was efficiently converted to amide $\mathbf{3 d g}$ (85\%). Introducing electron-withdrawing groups led to, respectively, $82 \%$ and $58 \%$ yield for $p-\mathrm{CO}_{2} \mathrm{Me}$ and $p-\mathrm{CF}_{3}$ groups. Surprisingly, $p-\mathrm{NO}_{2}$ substitution, which accounted for the best yield in coppercatalyzed amidation, gave a poor $47 \%$ of corresponding amide 3gg. When meta-substituted benzylic alcohols were used, yields from $65 \%$ to $89 \%$ were obtained, with methoxy (3hg), chloro (3ig)

\begin{tabular}{|c|c|c|}
\hline & TON & $\mathrm{TOF}\left(\mathrm{h}^{-1}\right)$ \\
\hline $\mathrm{FeCl}_{2} \cdot 4 \mathrm{H}_{2} \mathrm{O} / \mathrm{TBHP}$ & $<8.9$ & $<2.2$ \\
\hline $\mathrm{CuO} / \mathrm{TBHP}$ (Lamaty) $)^{12}$ & $<44$ & $<11$ \\
\hline $\mathrm{ZnI}_{2} / \mathrm{TBHP}\left(\right.$ Beller) ${ }^{13}$ & $<17$ & $<1.1$ \\
\hline $\mathrm{Fe}\left(\mathrm{NO}_{3}\right)_{3} \cdot 9 \mathrm{H}_{2} \mathrm{O} / \mathrm{TEMPO} / \mathrm{TBHP}(\text { Chen })^{15}$ & $<16.2$ & $<0.9$ \\
\hline $\mathrm{FeCl}_{3} \cdot 6 \mathrm{H}_{2} \mathrm{O} / \mathrm{NCS} / \mathrm{TBHP}(\text { De Luca) })^{14}$ & $<7$ & n.d. ${ }^{b}$ \\
\hline
\end{tabular}
and nitro (3jg) substituents. Finally, ortho substitution, which combined steric and electronic influence, gave lower yields of amides $3 \mathbf{k g}$ and $3 \mathbf{l g}$, bearing, respectively, methoxy (45\%) and chloro groups (41\%). ortho-Nitrobenzyl alcohol did not react as expected and amide 3mg was not observed.

In order to compare this catalytic reaction with literature, TON (turnover number) and TOF (turnover frequency) were calculated using the best results reported in previous works and herein (Table 5). The copper-catalyzed reaction ${ }^{12}$ was by far the most efficient regarding TON (up to 44) and TOF (up to $11 \mathrm{~h}^{-1}$ ), as only $2 \mathrm{~mol} \%$ of $\mathrm{CuO}$ and $4 \mathrm{~h}$ of reaction were necessary to obtain good yields. Apart from copper, considering TOF, the use of $\mathrm{FeCl}_{2} \cdot 4 \mathrm{H}_{2} \mathrm{O}$ as catalyst (TOF up to $2.2 \mathrm{~h}^{-1}$ ) gave better results than other catalytic systems involving zinc or iron (TOF up to $1.1 \mathrm{~h}^{-1}$ ), therefore attesting that these reaction conditions represent a good alternative to reported procedures.

Table 5

Comparison of TON and TOF with literature ${ }^{\mathrm{a}}$

a $\mathrm{TON}=(\mathrm{mmol}$ of product $) /(\mathrm{mmol}$ of catalyst $) . \mathrm{TOF}=\mathrm{TON} /($ reaction time $)$. TON and TOF were calculated using literature data.

b Reaction time was not specified in the publication.

Control experiments were then run to assess if the ironcatalyzed amidation was indeed going through the general mechanism depicted in Scheme 1. First, when benzoic acid was used instead of benzylic alcohol in the presence of cyclohexylamine and under the exact optimized conditions used above, no reaction occurred, proving that no direct thermal condensation between acid and amine happened as described elsewhere (Scheme 3, Eq. 1).$^{17}$ In addition, when aldimine $\mathbf{4}$ was reacted under the amidation conditions, no expected amide was observed, proving that formation of amide bond might result from oxidation of the hemiaminal rather than from corresponding aldimine (Scheme 3, Eq. 2). Finally, reaction between benzylic alcohol and cyclohexylamine was performed in the presence of TEMPO or hydroquinone as radical scavengers (Scheme 3, Eq. 3). Expected amide 3aa could not be observed under those conditions, indicating that the iron-catalyzed amidation might certainly involve the formation of radical species during the oxidation process. However, no trapping intermediate could be isolated.

$$
\begin{aligned}
& \text { (1) } \mathrm{Ph} \stackrel{\text { 兵 }}{\mathrm{OH}}+\stackrel{\oplus}{\mathrm{H}_{3} \mathrm{~N}-\mathrm{Cy}} \quad \mathrm{FeCl}_{2} \cdot 4 \mathrm{H}_{2} \mathrm{O}(10 \mathrm{~mol} \%)
\end{aligned}
$$

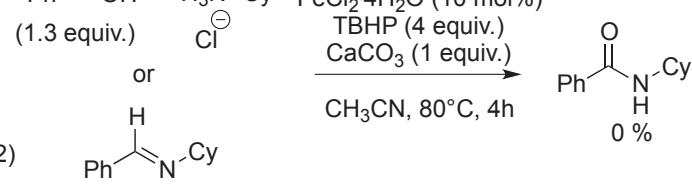

$$
\begin{aligned}
& \text { (2) } \\
& 4 \\
& \mathrm{FeCl}_{2} \cdot 4 \mathrm{H}_{2} \mathrm{O}(10 \mathrm{~mol} \%) \\
& \text { TBHP (4 equiv.) }
\end{aligned}
$$

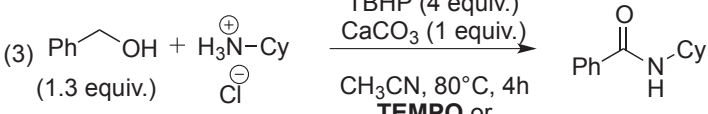

$$
\begin{aligned}
& \text { TEMPO or } \\
& \text { hydroquinone (4 equiv.) } \\
& 0 \%
\end{aligned}
$$

Scheme 3. Control experiments for amidation reaction. 
To prove that this methodology could be efficiently applied to the synthesis of compounds of interest, the synthesis of moclobemide was envisioned using an iron-catalyzed amidation as a first synthetic step (Scheme 4). Moclobemide, an Hoffmann-La Roche drug used against depression and social anxiety, could be readily obtained from 4-chloro- $N$-(2-chloroethyl)benzamide 5a. Thus, $p$ chlorobenzyl alcohol 1d was reacted with commercially available 2-chloroethylamine hydrochloride. The isolated yield of amide $\mathbf{5 a}$ being only $35 \%$, the synthesis of synthetic intermediates $\mathbf{5 b}$ and $\mathbf{5 c}$ was intended. While reacting $\mathbf{1 d}$ with ethanolamine hydrochloride gave also a poor yield (5b, 30\%), the use of glycine methylester yielded amide $5 \mathbf{c}$ in $79 \%$ yield on a $0.5 \mathrm{mmol}$ scale. On a 10 -fold scale $(5 \mathrm{mmol})$ under the exact same conditions, amide $\mathbf{5 c}$ could be isolated with a slight depletion of the yield (57\%). The final steps of the synthesis were achieved according to literature procedures. Reacting $\mathrm{NaBH}_{4}$ in a mixture $\mathrm{THF} / \mathrm{MeOH}^{18}$ allowed the reduction of 5c to alcohol 5b in $89 \%$ yield. Quantitative substitution of the hydroxyl group by a chlorine atom and reaction with morpholine directly yielded moclobemide, with a $42 \%$ yield over two-steps. This modest yield was consistent with the reported one (33\%), ${ }^{19}$ and moclobemide could be obtained from easily available substrates and in a more sustainable manner to generate the amide functionality.

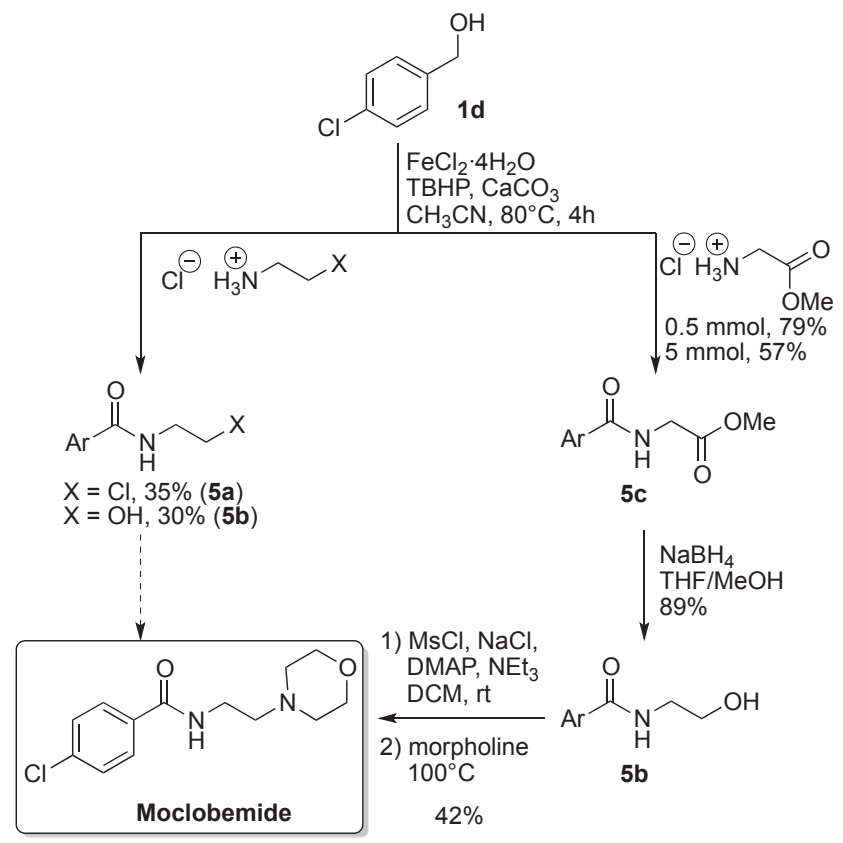

Scheme 4. Synthesis of moclobemide using iron-catalyzed amidation.

\section{Conclusions}

In conclusion, we described a new protocol of iron-catalyzed direct formation of benzamides from alcohols and amines. This system required the use of a cheap iron salt and TBHP as a non-toxic stoichiometric oxidant, which decomposes only to form tert-butanol and water. Ammonium salts of amines, which are easily accessible, user-friendly and stable, were used in this study. Primary, secondary aliphatic and benzylic amines were well tolerated, as well as benzylic alcohols featuring electron-withdrawing and -donating groups. To validate the usefulness of this methodology, the synthesis of the anti-anxiety drug moclobemide was performed starting from easily available substrates.

\section{Experimental section}

\subsection{General}

In a sealed tube were added $\mathrm{FeCl}_{2} \cdot 4 \mathrm{H}_{2} \mathrm{O}(9.9 \mathrm{mg}, 0.05 \mathrm{mmol}$, $10 \mathrm{~mol} \%)$, cyclohexylamine $\cdot \mathrm{HCl}(67.8 \mathrm{mg}, 0.5 \mathrm{mmol}, 1$ equiv) and $\mathrm{CaCO}_{3}$ (50.1 mg, $0.5 \mathrm{mmol}, 1$ equiv). $1 \mathrm{~mL}$ of $\mathrm{CH}_{3} \mathrm{CN}$, benzyl alcohol (67 $\mu \mathrm{L}, 0.65 \mathrm{mmol}, 1.3$ equiv) and tert-butylhydroperoxide (70\% in $\mathrm{H}_{2} \mathrm{O}, 140 \mu \mathrm{L}, 2$ equiv) were then added. After $2 \mathrm{~h}$ of stirring at $80^{\circ} \mathrm{C}$, another 2 equiv of tert-butylhydroperoxide $\left(70 \%\right.$ in $\left.\mathrm{H}_{2} \mathrm{O}\right)$ were added, and the mixture was further stirred for $2 \mathrm{~h}$. After cooling to $\mathrm{rt}, \mathrm{HCl} 1 \mathrm{~N}(5 \mathrm{~mL})$ and AcOEt $(5 \mathrm{~mL})$ were added. The mixture was extracted with AcOEt $(2 \times 5 \mathrm{~mL})$, and the combined organic phases were washed with saturated aqueous $\mathrm{NaHCO}_{3}$ solution $(10 \mathrm{~mL})$, brine $(10 \mathrm{~mL})$, dried over magnesium sulfate and concentrated under reduced pressure. The crude mixture was purified by chromatography on silica gel using mixtures of cyclohexane and ethyl acetate as eluant.

4.1.1. $N$-Cyclohexylbenzamide 3 aa. $^{20} 89 \%$ yield $(90.2 \mathrm{mg})$ as a white solid. ${ }^{1} \mathrm{H}$ NMR $\left(\mathrm{CDCl}_{3}, 200 \mathrm{MHz}\right): \delta(\mathrm{ppm})=7.85-7.64(\mathrm{~m}, 2 \mathrm{H})$, $7.53-7.30(\mathrm{~m}, 3 \mathrm{H}), 6.17(\mathrm{~d}, 1 \mathrm{H}, J=6.6 \mathrm{~Hz}), 3.95(\mathrm{ttd}, 1 \mathrm{H}, J=11.9,8.0$, $3.8 \mathrm{~Hz}), 2.09-1.91(\mathrm{~m}, 2 \mathrm{H}), 1.84-1.55(\mathrm{~m}, 3 \mathrm{H}), 1.50-1.10(\mathrm{~m}, 5 \mathrm{H}) .{ }^{13} \mathrm{C}$ $\operatorname{NMR}\left(\mathrm{CDCl}_{3}, 75 \mathrm{MHz}\right): \delta(\mathrm{ppm})=166.8,135.2,134.3,128.6,127.0$, 48.8, 33.3, 25.7, 25.0.

4.1.2. $\mathrm{N}$-Methylbenzamide $3 \boldsymbol{a b}$. $70 \%$ yield $(48.7 \mathrm{mg}$ ) as a white solid. ${ }^{1} \mathrm{H}$ NMR $\left(\mathrm{CDCl}_{3}, 200 \mathrm{MHz}\right): \delta(\mathrm{ppm})=7.84-7.67(\mathrm{~m}, 2 \mathrm{H})$, $7.52-7.30(\mathrm{~m}, 3 \mathrm{H}), 6.71(\mathrm{~s}, 1 \mathrm{H}), 2.96(\mathrm{~d}, J=4.8 \mathrm{~Hz}, 3 \mathrm{H}) .{ }^{13} \mathrm{C}$ NMR $\left(\mathrm{CDCl}_{3}, 75 \mathrm{MHz}\right): \delta(\mathrm{ppm})=168.5,134.7,131.4,128.6,127.0,26.9$.

4.1.3. $N$-n-Butylbenzamide 3ac. ${ }^{21} 76 \%$ yield $(67.4 \mathrm{mg})$ as a white solid. ${ }^{1} \mathrm{H}$ NMR $\left(\mathrm{CDCl}_{3}, 200 \mathrm{MHz}\right): \delta(\mathrm{ppm})=7.78-7.73(\mathrm{~m}, 2 \mathrm{H})$, 7.46-7.34 (m, 3H), 6.42 (br s, $1 \mathrm{H}), 3.42(\mathrm{q}, 2 \mathrm{H}, J=6.5 \mathrm{~Hz}), 1.65-1.47$ $(\mathrm{m}, 2 \mathrm{H}), 1.43-1.24(\mathrm{~m}, 2 \mathrm{H}), 0.93(\mathrm{t}, 3 \mathrm{H}, J=7.2 \mathrm{~Hz}) .{ }^{13} \mathrm{C} \mathrm{NMR}\left(\mathrm{CDCl}_{3}\right.$, $75 \mathrm{MHz}): \delta(\mathrm{ppm})=167.7,134.9,131.3,128.6,127.0,39.9,31.8,20.2$, 13.9.

4.1.4. N-Iso-propylbenzamide 3 ad. $^{22} 72 \%$ yield $(58.6 \mathrm{mg})$ as a white solid. ${ }^{1} \mathrm{H}$ NMR $\left(\mathrm{CDCl}_{3}, 200 \mathrm{MHz}\right): \delta(\mathrm{ppm})=7.84-7.68(\mathrm{~m}, 1 \mathrm{H})$, 7.53-7.30 (m, 2H), $6.18(\mathrm{~s}, 1 \mathrm{H}), 4.40-4.11(\mathrm{~m}, 1 \mathrm{H}), 1.23(\mathrm{~d}, J=6.6 \mathrm{~Hz}$, $3 \mathrm{H}) .{ }^{13} \mathrm{C} \mathrm{NMR}\left(\mathrm{CDCl}_{3}, 75 \mathrm{MHz}\right): \delta(\mathrm{ppm})=166.8,135.1,131.3,128.5$, 126.9, 41.9, 22.9 .

4.1.5. N-tert-Butylbenzamide $3 \boldsymbol{a e} .^{10 d} 59 \%$ yield $(52.0 \mathrm{mg})$ as a white solid. ${ }^{1} \mathrm{H}$ NMR $\left(\mathrm{CDCl}_{3}, 200 \mathrm{MHz}\right): \delta(\mathrm{ppm})=7.93-7.60(\mathrm{~m}, 2 \mathrm{H}), 7.42$ $(\mathrm{m}, 3 \mathrm{H}), 5.99(\mathrm{~s}, 1 \mathrm{H}), 1.46(\mathrm{~s}, 9 \mathrm{H}) .{ }^{13} \mathrm{C} \mathrm{NMR}\left(\mathrm{CDCl}_{3}, 75 \mathrm{MHz}\right)$ : $\delta(\mathrm{ppm})=167.0,136.0,131.2,128.6,126.8,51.7,20.0$.

4.1.6. $\mathrm{N}$-Benzylbenzamide $3 \boldsymbol{a f f}^{23} 63 \%$ yield $(66.6 \mathrm{mg})$ as a white solid. ${ }^{1} \mathrm{H}$ NMR $\left(\mathrm{CDCl}_{3}, 200 \mathrm{MHz}\right): \delta(\mathrm{ppm})=7.75-7.70(\mathrm{~m}, 2 \mathrm{H})$, 7.47-7.17 (m, 8H), 6.62 (br s, $1 \mathrm{H}), 4.55$ (d, $2 \mathrm{H}, J=5.7 \mathrm{~Hz}) .{ }^{13} \mathrm{C}$ NMR $\left(\mathrm{CDCl}_{3}, 75 \mathrm{MHz}\right): \delta(\mathrm{ppm})=167.6,138.4,134.4,131.5,128.8,128.6$, 127.9, 127.5, 127.1, 44.1.

4.1.7. $N$-( $\alpha$-Methylbenzyl)benzamide 3ag. ${ }^{24} 77 \%$ yield $(86.2 \mathrm{mg})$ as a white solid. ${ }^{1} \mathrm{H}$ NMR $\left(\mathrm{CDCl}_{3}, 200 \mathrm{MHz}\right): \delta(\mathrm{ppm})=7.89-7.65(\mathrm{~m}$, $2 \mathrm{H}), 7.59-7.20(\mathrm{~m}, 8 \mathrm{H}), 6.56(\mathrm{~d}, J=7.1 \mathrm{~Hz}, 1 \mathrm{H}), 5.33$ (p, $J=7.0 \mathrm{~Hz}$, $1 \mathrm{H}), 1.59(\mathrm{~d}, J=6.9 \mathrm{~Hz}, 3 \mathrm{H}) .{ }^{13} \mathrm{C} \mathrm{NMR}\left(\mathrm{CDCl}_{3}, 75 \mathrm{MHz}\right): \delta(\mathrm{ppm})=$ $166.7,143.3,134.7,131.5,128.8,128.6,127.5,127.1,126.3,49.3$, 21.8 .

4.1.8. N-Benzoyl-phenylglycinol $3 \boldsymbol{a h h}^{25} 71 \%$ yield $(85.9 \mathrm{mg})$ as a white solid. ${ }^{1} \mathrm{H}$ NMR (DMSO- $\left.d_{6}, 200 \mathrm{MHz}\right): \delta(\mathrm{ppm})=8.70(\mathrm{~d}$, $J=8.1 \mathrm{~Hz}, 1 \mathrm{H}), 7.90(\mathrm{~d}, J=6.3 \mathrm{~Hz}, 2 \mathrm{H}), 7.64-7.11(\mathrm{~m}, 8 \mathrm{H}), 5.07$ (dd, $J=13.8,8.1 \mathrm{~Hz}, 1 \mathrm{H}), 4.94(\mathrm{t}, J=5.8 \mathrm{~Hz}, 1 \mathrm{H}), 3.80-3.55(\mathrm{~m}, 2 \mathrm{H}) .{ }^{13} \mathrm{C}$ 
NMR (DMSO- $\left.d_{6}, 75 \mathrm{MHz}\right): \delta(\mathrm{ppm})=166.3,141.4,134.7,131.2,128.3$, 128.2, 127.4, 127.0, 126.9, 64.6, 56.1.

4.1.9. $\mathrm{N}$-(1,2-Diphenylethyl)benzamide $3 \boldsymbol{a i}^{26}{ }^{26} 52 \%$ yield (78.4 mg) as a white solid. ${ }^{1} \mathrm{H}$ NMR $\left(\mathrm{CDCl}_{3}, 200 \mathrm{MHz}\right): \delta(\mathrm{ppm})=7.75-7.55(\mathrm{~m}$, $2 \mathrm{H}), 7.51-7.13(\mathrm{~m}, 11 \mathrm{H}), 7.08(\mathrm{~d}, J=5.9 \mathrm{~Hz}, 2 \mathrm{H}), 6.56(\mathrm{~d}, J=7.4 \mathrm{~Hz}, 1 \mathrm{H})$, $5.45(\mathrm{q}, J=7.2 \mathrm{~Hz}, 1 \mathrm{H}), 3.19(\mathrm{~d}, J=7.0 \mathrm{~Hz}, 2 \mathrm{H}) .{ }^{13} \mathrm{C} \mathrm{NMR}\left(\mathrm{CDCl}_{3}\right.$, $75 \mathrm{MHz}): \delta(\mathrm{ppm})=166.9,141.6,137.35,134.7,131.55,129.5,128.7$, 128.6, 128.5, 127.6, 127.0, 126.8, 54.9, 42.7.

4.1.10. N-Phenethylbenzamide $3 \boldsymbol{a j}^{10 a, 27} 61 \%$ yield $(69.0 \mathrm{mg})$ as a white solid. ${ }^{1} \mathrm{H} \mathrm{NMR}\left(\mathrm{CDCl}_{3}, 200 \mathrm{MHz}\right): \delta(\mathrm{ppm})=7.77-7.60(\mathrm{~m}$, $2 \mathrm{H}), 7.54-7.16(\mathrm{~m}, 8 \mathrm{H}), 6.23(\mathrm{~s}, 1 \mathrm{H}), 3.72(\mathrm{q}, 2 \mathrm{H}, J=6.9 \mathrm{~Hz}), 2.94(\mathrm{t}$, $2 \mathrm{H}, J=6.9 \mathrm{~Hz}) .{ }^{13} \mathrm{C}$ NMR $\left(\mathrm{CDCl}_{3}, 75 \mathrm{MHz}\right): \delta(\mathrm{ppm})=167.6,139.0$, 134.7, 131.5, 128.9, 128.8, 128.6, 126.9, 126.7, 41.3, 35.8.

4.1.11. N-p-Methoxyphenethylbenzamide 3ak ${ }^{28} 58 \%$ yield (73.8 mg) as a white solid. ${ }^{1} \mathrm{H}$ NMR $\left(\mathrm{CDCl}_{3}, 200 \mathrm{MHz}\right): \delta(\mathrm{ppm})=7.72-7.68(\mathrm{~m}$, $2 \mathrm{H}), 7.51-7.34(\mathrm{~m}, 3 \mathrm{H}), 7.16-7.12(\mathrm{~m}, 2 \mathrm{H}), 6.87-6.83(\mathrm{~m}, 2 \mathrm{H}), 6.36$ (br s, $1 \mathrm{H}), 3.78(\mathrm{~s}, 3 \mathrm{H}), 3.66(\mathrm{q}, 2 \mathrm{H}, J=7.0 \mathrm{~Hz}), 2.86(\mathrm{t}, 2 \mathrm{H}, J=7.0 \mathrm{~Hz})$. ${ }^{13} \mathrm{C} \mathrm{NMR}\left(\mathrm{CDCl}_{3}, 75 \mathrm{MHz}\right): \delta(\mathrm{ppm})=167.6,158.4,134.8,131.4,131.0$, $129.8,128.6,127.9,126.9,114.2,55.3,41.4,34.9$.

4.1.12. N-Benzoylphenylalanine tert-butyl ester $3 \mathbf{a l}^{29} 77 \%$ yield $(124.8 \mathrm{mg})$ as a white solid. ${ }^{1} \mathrm{H} \mathrm{NMR}\left(\mathrm{CDCl}_{3}, 200 \mathrm{MHz}\right): \delta(\mathrm{ppm})=$ 7.72-7.55 (m, 2H), 7.46-7.23 (m, 3H), 7.23-7.05 (m, 5H), 6.67 (d, $1 \mathrm{H}, J=7.3 \mathrm{~Hz}), 4.88(\mathrm{dt}, 1 \mathrm{H}, J=7.4,5.8 \mathrm{~Hz}), 3.14(\mathrm{~d}, 2 \mathrm{H}, J=5.7 \mathrm{~Hz}), 1.34$ $(\mathrm{s}, 9 \mathrm{H}) \cdot{ }^{13} \mathrm{C}$ NMR $\left(\mathrm{CDCl}_{3}, 75 \mathrm{MHz}\right): \delta(\mathrm{ppm})=170.8,166.8,136.3$, 134.1, 131.7, 129.6, 128.6, 128.4, 127.0, 82.6, 54.0, 38.1, 28.0.

4.1.13. $\mathrm{N}$-Benzoylphenylalanine $\cdot 1 / 2 \mathrm{H}_{2} \mathrm{O}$ 3am. $70 \%$ yield $(97.6 \mathrm{mg})$ as a white solid. ${ }^{1} \mathrm{H} \mathrm{NMR}\left(\mathrm{CDCl}_{3}, 200 \mathrm{MHz}\right): \delta(\mathrm{ppm})=7.73(\mathrm{~d}, J=6.8 \mathrm{~Hz}$, $2 \mathrm{H}), 7.64-7.28(\mathrm{~m}, 8 \mathrm{H}), 6.87(\mathrm{~s}, 1 \mathrm{H}, \mathrm{NH}), 5.81\left(\mathrm{~s}, 1 \mathrm{H}, \mathrm{CO}_{2} \mathrm{H}\right), 5.41(\mathrm{~s}$, $\left.1 \mathrm{H}, 1 / 2 \mathrm{H}_{2} \mathrm{O}\right), 4.90(\mathrm{~s}, 1 \mathrm{H}), 3.40-3.01(\mathrm{~m}, 2 \mathrm{H}) .{ }^{13} \mathrm{C}$ NMR $\left(\mathrm{CD}_{3} \mathrm{OD}\right.$, $75 \mathrm{MHz}): \delta(\mathrm{ppm})=176.3,170.1,138.7,135.3,132.8,130.3,129.5$, 129.4, 128.4, 127.8, 56.3, 38.9.

4.1.14. $\mathrm{N}, \mathrm{N}$-Dibenzylbenzamide $\mathbf{3 a n}^{29} 69 \%$ yield $(104.0 \mathrm{mg}$ ) as a white solid. ${ }^{1} \mathrm{H}$ NMR $\left(\mathrm{CDCl}_{3}, 200 \mathrm{MHz}\right): \delta(\mathrm{ppm})=7.65-7.29(\mathrm{~m}$, $7 \mathrm{H}), 7.18(\mathrm{~s}, 1 \mathrm{H}), 4.73(\mathrm{~s}, 1 \mathrm{H}), 4.42(\mathrm{~s}, 1 \mathrm{H}) .{ }^{13} \mathrm{C} \mathrm{NMR}\left(\mathrm{CDCl}_{3}, 75 \mathrm{MHz}\right)$ : $\delta(\mathrm{ppm})=172.3,137.0,136.5,136.2,129.7,128.9,128.6,128.5,127.6$, 127.1, 126.8, 51.6, 46.9.

4.1.15. $\mathrm{N}$-Benzoyl proline tert-butyl ester 3 ao. $55 \%$ yield $(76.4 \mathrm{mg})$ as a white solid. ${ }^{1} \mathrm{H}$ NMR $\left(\mathrm{CDCl}_{3}, 200 \mathrm{MHz}\right): \delta(\mathrm{ppm})=7.56-7.51(\mathrm{~m}$, $2 \mathrm{H}), 7.42-7.29(\mathrm{~m}, 3 \mathrm{H}), 4.52$ (dd, $J=8.0,5.5 \mathrm{~Hz}, 1 \mathrm{H}, 70 \%), 4.26-4.13$ (m, 1H, 30\%), 3.79-3.41 (m, 2H), 2.34-2.14 (m, 1H), 2.01-1.79 (m,

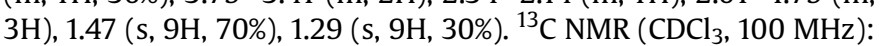
$\delta(\mathrm{ppm})=171.5,169.6,136.6,130.1,128.3,127.3,126.8,81.3,60.0$, 50.0, 29.5, 28.1, 27.8, 25.4 (major rotamer). $\delta(\mathrm{ppm})=171.5,170.5$, 137.2, 129.7, 81.8, 62.1, 46.7, 31.6, 29.8, 22.7 (minor rotamer).

4.1.16. $\mathrm{N}$-Benzoyl piperidine 3 ap. $^{7} 66 \%$ yield $(62.2 \mathrm{mg})$ as a translucid oil. ${ }^{1} \mathrm{H}$ NMR $\left(\mathrm{CDCl}_{3}, 200 \mathrm{MHz}\right): \delta(\mathrm{ppm})=7.36(\mathrm{~s}, 5 \mathrm{H}), 3.68(\mathrm{~s}$, $2 \mathrm{H}), 3.31(\mathrm{~s}, 2 \mathrm{H}), 1.64-1.51(\mathrm{~m}, 6 \mathrm{H}) .{ }^{13} \mathrm{C} \mathrm{NMR}\left(\mathrm{CDCl}_{3}, 75 \mathrm{MHz}\right)$ : $\delta(\mathrm{ppm})=170.3,136.6,129.4,128.4,126.8,48.8,43.1,26.6,25.7,24.6$.

4.1.17. N-Benzoyl morpholine 3aq. ${ }^{10 c} 64 \%$ yield $(61.2 \mathrm{mg})$ as a white oil. ${ }^{1} \mathrm{H}$ NMR $\left(\mathrm{CDCl}_{3}, 200 \mathrm{MHz}\right): \delta(\mathrm{ppm})=7.39(\mathrm{~s}, 5 \mathrm{H}), 3.69(\mathrm{br} \mathrm{s}, 6 \mathrm{H})$, 3.45 (br s, $2 \mathrm{H}) .{ }^{13} \mathrm{C} \mathrm{NMR}\left(\mathrm{CDCl}_{3}, 75 \mathrm{MHz}\right): \delta(\mathrm{ppm})=170.5,135.4$, 129.9, 128.6, 127.1, 67.0.

4.1.18. $N$-(p-Methoxybenzoyl)- $\alpha$-methylbenzyl amine 3bg. $^{30} 58 \%$ yield $(74.4 \mathrm{mg})$ as a white solid. ${ }^{1} \mathrm{H}$ NMR $\left(\mathrm{CDCl}_{3}, 200 \mathrm{MHz}\right)$ : $\delta(\mathrm{ppm})=7.71(\mathrm{~d}, 2 \mathrm{H}, J=8.9 \mathrm{~Hz}), 7.47-7.08(\mathrm{~m}, 6 \mathrm{H}), 6.95-6.79(\mathrm{~m}$, 2H), $6.45(\mathrm{~d}, 1 \mathrm{H}, J=7.5 \mathrm{~Hz}), 5.28(\mathrm{p}, 1 \mathrm{H}, J=7.0 \mathrm{~Hz}), 3.78(\mathrm{~s}, 3 \mathrm{H}), 1.54$ $(\mathrm{d}, 3 \mathrm{H}, J=6.9 \mathrm{~Hz}) .{ }^{13} \mathrm{C} \mathrm{NMR}\left(\mathrm{CDCl}_{3}, 75 \mathrm{MHz}\right): \delta(\mathrm{ppm})=166.3,162.2$, 143.5, 128.9, 128.8, 127.4, 126.9, 126.3, 113.8, 55.5, 49.2, 21.9.

4.1.19. $N$-(p-Methylbenzoyl)- $\alpha$-methylbenzyl amine 3 cg. $^{31} 69 \%$ yield $(83.8 \mathrm{mg})$ as a white solid. ${ }^{1} \mathrm{H}$ NMR $\left(\mathrm{CDCl}_{3}, 200 \mathrm{MHz}\right): \delta(\mathrm{ppm})=7.65$ $(\mathrm{d}, 2 \mathrm{H}, J=8.2 \mathrm{~Hz}), 7.44-7.11(\mathrm{~m}, 7 \mathrm{H}), 6.37(\mathrm{~d}, 1 \mathrm{H}, J=7.0 \mathrm{~Hz}), 5.31(\mathrm{p}$, $1 \mathrm{H}, J=7.0 \mathrm{~Hz}), 2.36(\mathrm{~s}, 3 \mathrm{H}), 1.57(\mathrm{~d}, 3 \mathrm{H}, J=6.9 \mathrm{~Hz}) .{ }^{13} \mathrm{C} \mathrm{NMR}\left(\mathrm{CDCl}_{3}\right.$, $75 \mathrm{MHz}): \delta(\mathrm{ppm})=166.7,143.4,142.0,131.8,129.3,128.8,127.5$, 127.1, 126.4, 49.2, 21.9, 21.5.

4.1.20. $\mathrm{N}$-(p-Chlorobenzoyl)- $\alpha$-methylbenzyl amine $3 \mathbf{d g}^{32} 85 \%$ yield $(111.4 \mathrm{mg})$ as a white solid. ${ }^{1} \mathrm{H}$ NMR $\left(\mathrm{CDCl}_{3}, 200 \mathrm{MHz}\right): \delta(\mathrm{ppm})=$ $7.61(\mathrm{dd}, 2 \mathrm{H}, J=8.2,1.2 \mathrm{~Hz}), 7.34-7.13(\mathrm{~m}, 7 \mathrm{H}), 6.59(\mathrm{~d}, 1 \mathrm{H}, J=7.2 \mathrm{~Hz})$, 5.21 (p, $1 \mathrm{H}, J=7.0 \mathrm{~Hz}), 1.50$ (dd, $3 \mathrm{H}, J=6.9,1.0 \mathrm{~Hz}) .{ }^{13} \mathrm{C} \mathrm{NMR}\left(\mathrm{CDCl}_{3}\right.$, $75 \mathrm{MHz}): \delta(\mathrm{ppm})=165.7,143.1,137.7,133.0,128.8,128.8,128.6$, 127.6, 126.3, 49.5, 21.8 .

4.1.21. $N$-(p-(Methoxycarbonyl)benzoyl)- $\alpha$-methylbenzyl amine 3eg. ${ }^{31} 82 \%$ yield (115.5 mg) as a white solid. ${ }^{1} \mathrm{H}$ NMR $\left(\mathrm{CDCl}_{3}\right.$, $200 \mathrm{MHz}): \delta(\mathrm{ppm})=8.09(\mathrm{~d}, J=8.6 \mathrm{~Hz}, 2 \mathrm{H}), 7.82(\mathrm{~d}, J=8.6 \mathrm{~Hz}, 2 \mathrm{H})$, 7.45-7.28 (m, 5H), 6.34 (d, $J=6.7 \mathrm{~Hz}, 1 \mathrm{H}), 5.35$ (p, $J=6.8 \mathrm{~Hz}, 1 \mathrm{H})$, $1.63(\mathrm{~d}, J=6.9 \mathrm{~Hz}, 3 \mathrm{H}) .{ }^{13} \mathrm{C}$ NMR $\left(\mathrm{CDCl}_{3}, 75 \mathrm{MHz}\right): \delta(\mathrm{ppm})=166.4$, $165.9,143.0,138.6,132.6,129.8,128.8,127.6,127.2,126.3,52.4$, 49.5, 21.7.

4.1.22. $N$-(p-Trifluorobenzoyl)- $\alpha$-methylbenzyl amine 3 fg. $58 \%$ yield $(85.2 \mathrm{mg})$ as a white solid. ${ }^{1} \mathrm{H}$ NMR $\left(\mathrm{CDCl}_{3}, 200 \mathrm{MHz}\right): \delta(\mathrm{ppm})=7.83$ (d, $J=8.2 \mathrm{~Hz}, 2 \mathrm{H}), 7.60$ (d, $J=8.3 \mathrm{~Hz}, 2 \mathrm{H}), 7.42-7.26$ (m, 5H), 6.85 (d, $J=7.6 \mathrm{~Hz}, 1 \mathrm{H}), 5.30(\mathrm{p}, J=7.0 \mathrm{~Hz}, 1 \mathrm{H}), 1.59(\mathrm{~d}, J=6.9 \mathrm{~Hz}, 3 \mathrm{H}) .{ }^{13} \mathrm{C} \mathrm{NMR}$ $\left(\mathrm{CDCl}_{3}, 75 \mathrm{MHz}\right): \delta(\mathrm{ppm})=165.6,142.9,138.0,133.2(\mathrm{q}, J=32.5 \mathrm{~Hz})$, 128.9, 127.7, 127.6, 126.3, 125.7, 125.6, 123.8 (q, $J=271 \mathrm{~Hz}), 49.6$, 21.7. ${ }^{19} \mathrm{~F} \mathrm{NMR}\left(\mathrm{CDCl}_{3}, 282 \mathrm{MHz}\right): \delta(\mathrm{ppm})=-62.9$. HRMS $\left(\mathrm{ESI}^{+}\right) \mathrm{m} / z$ calculated for $\left(\mathrm{MH}^{+}\right)$: 294.1106. Found: 294.1107.

4.1.23. $N$-(p-Trifluorobenzoyl)- $\alpha$-methylbenzyl amine $3 g$. $^{33} 53 \%$ yield $(71.8 \mathrm{mg})$ as a white solid. ${ }^{1} \mathrm{H}$ NMR $\left(\mathrm{CDCl}_{3}, 200 \mathrm{MHz}\right)$ : $\delta(\mathrm{ppm})=8.31-8.12(\mathrm{~m}, 2 \mathrm{H}), 7.98-7.83(\mathrm{~m}, 2 \mathrm{H}), 7.44-7.27(\mathrm{~m}, 5 \mathrm{H})$, $6.70(\mathrm{~d}, 1 \mathrm{H}, J=7.3 \mathrm{~Hz}), 5.30$ (p, 1H, $J=7.0 \mathrm{~Hz}), 1.61(\mathrm{~d}, 3 \mathrm{H}, J=6.9 \mathrm{~Hz})$. ${ }^{13} \mathrm{C} \mathrm{NMR}\left(\mathrm{CDCl}_{3}, 75 \mathrm{MHz}\right): \delta(\mathrm{ppm})=164.8,149.5,142.7,140.2,128.9$, $128.3,127.8,126.3,123.7,49.8,21.7$.

4.1.24. $N$-(m-Methoxybenzoyl)- $\alpha$-methylbenzyl amine $3 \mathbf{h g} .{ }^{12} 89 \%$ yield $(114.5 \mathrm{mg})$ as a white solid. ${ }^{1} \mathrm{H}$ NMR $\left(\mathrm{CDCl}_{3}, 200 \mathrm{MHz}\right)$ : $\delta(\mathrm{ppm})=7.43-7.34(\mathrm{~m}, 8 \mathrm{H}), 7.21-6.92(\mathrm{~m}, 1 \mathrm{H}), 6.65(\mathrm{~d}, J=6.6 \mathrm{~Hz}$, $1 \mathrm{H}), 5.56-5.23(\mathrm{p}, 1 \mathrm{H}, J=7.0 \mathrm{~Hz}), 3.86(\mathrm{~s}, 3 \mathrm{H}), 1.64(\mathrm{~d}, 3 \mathrm{H}, J=6.9 \mathrm{~Hz})$. ${ }^{13} \mathrm{C} \mathrm{NMR}\left(\mathrm{CDCl}_{3}, 75 \mathrm{MHz}\right): \delta(\mathrm{ppm})=166.5,159.9,143.3,136.1,129.6$, $128.8,127.5,126.3,118.8,117.7,112.5,55.5,49.4,21.8$.

4.1.25. $\mathrm{N}$-(m-Chloro)- $\alpha$-methylbenzyl amine $\mathbf{3 i g}^{34}$ 77\% yield $(100.2 \mathrm{mg})$ as a white solid. ${ }^{1} \mathrm{H}$ NMR $\left(\mathrm{CDCl}_{3}, 200 \mathrm{MHz}\right): \delta(\mathrm{ppm})=$ $7.72(\mathrm{t}, J=1.8 \mathrm{~Hz}, 1 \mathrm{H}), 7.64-7.54(\mathrm{~m}, 1 \mathrm{H}), 7.45-7.17(\mathrm{~m}, 7 \mathrm{H}), 6.74(\mathrm{~d}$, $J=7.5 \mathrm{~Hz}, 1 \mathrm{H}), 5.26(\mathrm{p}, J=7.0 \mathrm{~Hz}, 1 \mathrm{H}), 1.54(\mathrm{~d}, J=6.9 \mathrm{~Hz}, 3 \mathrm{H}) .{ }^{13} \mathrm{C} \mathrm{NMR}$ $\left(\mathrm{CDCl}_{3}, 75 \mathrm{MHz}\right): \delta(\mathrm{ppm})=165.5,143.0,136.5,134.7,131.5,129.9$, 128.8, 127.6, 127.4, 126.3, 125.2, 49.5, 21.7.

4.1.26. $\mathrm{N}$-(m-Nitro)- $\alpha$-methylbenzyl amine 3jg. $^{35} 65 \%$ yield $(88.0 \mathrm{mg})$ as a white solid. ${ }^{1} \mathrm{H} \mathrm{NMR}\left(\mathrm{CDCl}_{3}, 200 \mathrm{MHz}\right): \delta(\mathrm{ppm})=8.55$ $(\mathrm{s}, 1 \mathrm{H}), 8.25(\mathrm{~d}, J=8.1 \mathrm{~Hz}, 1 \mathrm{H}), 8.10(\mathrm{~d}, J=7.7 \mathrm{~Hz}, 1 \mathrm{H}), 7.52(\mathrm{t}, J=8.0 \mathrm{~Hz}$, 1H), 7.35-7.21 (m, 4H), $7.12(\mathrm{~d}, J=7.1 \mathrm{~Hz}, 1 \mathrm{H}), 5.28(\mathrm{p}, J=6.9 \mathrm{~Hz}, 1 \mathrm{H})$, $1.58(\mathrm{~d}, J=6.9 \mathrm{~Hz}, 3 \mathrm{H}) .{ }^{13} \mathrm{C} \mathrm{NMR}\left(\mathrm{CDCl}_{3}, 75 \mathrm{MHz}\right): \delta(\mathrm{ppm})=164.4$, 148.1, 142.8, 136.2, 133.5, 129.8, 128.8, 127.6, 126.3, 126.0, 122.0, 49.9, 21.7.

4.1.27. $\mathrm{N}$-(o-Methoxybenzoyl)- $\alpha$-methylbenzyl amine $\mathbf{3 k g .}{ }^{12} 45 \%$ yield $(57.4 \mathrm{mg})$ as a slurry. ${ }^{1} \mathrm{H} \mathrm{NMR}\left(\mathrm{CDCl}_{3}, 200 \mathrm{MHz}\right): \delta(\mathrm{ppm})=8.27$ (d, $1 \mathrm{H}, J=6.7 \mathrm{~Hz}$ ), 8.20 (dd, 1H, $J=7.8,1.8 \mathrm{~Hz}$ ), 7.55-7.18 (m, 6H), 7.08 
(dt, $1 \mathrm{H}, J=7.5,0.8 \mathrm{~Hz}), 6.97(\mathrm{~d}, 1 \mathrm{H}, J=8.3 \mathrm{~Hz}), 5.35$ (p, $1 \mathrm{H}, J=6.9 \mathrm{~Hz}$ ), $3.94(\mathrm{~s}, 3 \mathrm{H}), 1.59(\mathrm{~d}, J=6.9 \mathrm{~Hz}, 3 \mathrm{H}) .{ }^{13} \mathrm{C} \mathrm{NMR}\left(\mathrm{CDCl}_{3}, 75 \mathrm{MHz}\right)$ : $\delta(\mathrm{ppm})=164.7,157.6,143.8,133.0,132.4,128.8,127.3,126.2,121.5$, 111.5, 56.1, 49.3, 22.6 .

4.1.28. $N$-(o-Chlorobenzoyl)- $\alpha$-methylbenzyl amine $3 \mathbf{l g}{ }^{36} 41 \%$ yield (53.4 mg) as a white solid. ${ }^{1} \mathrm{H} \mathrm{NMR}\left(\mathrm{CDCl}_{3}, 200 \mathrm{MHz}\right): \delta(\mathrm{ppm})=7.65$ (dd, $J=6.0,3.1 \mathrm{~Hz}, 1 \mathrm{H}), 7.45-7.26(\mathrm{~m}, 8 \mathrm{H}), 6.46(\mathrm{~s}, 1 \mathrm{H}), 5.34(\mathrm{p}$, $J=7.0 \mathrm{~Hz}, 1 \mathrm{H}), 1.62$ (d, $J=6.9 \mathrm{~Hz}, 3 \mathrm{H}) .{ }^{13} \mathrm{C} \mathrm{NMR}\left(\mathrm{CDCl}_{3}, 75 \mathrm{MHz}\right)$ : $\delta(\mathrm{ppm})=165.6,142.9,135.2,131.4,130.8,130.4,130.3,128.9,127.6$, 127.2, 126.4, 49.8, 21.9.

4.1.29. $N$-(p-Chlorobenzoyl)-glycine methylester $5 \boldsymbol{5 c}^{10 e} 79 \%$ yield $(90.2 \mathrm{mg})$ as a white solid. ${ }^{1} \mathrm{H}$ NMR $\left(\mathrm{CDCl}_{3}, 200 \mathrm{MHz}\right): \delta(\mathrm{ppm})=$ 7.82-7.68 (m, 2H), 7.48-7.36 (m, 2H), $6.64(\mathrm{~s}, 1 \mathrm{H}), 4.24(\mathrm{~d}, J=5.0 \mathrm{~Hz}$, $2 \mathrm{H}), 3.81(\mathrm{~s}, 3 \mathrm{H}) .{ }^{13} \mathrm{C} \mathrm{NMR}\left(\mathrm{CDCl}_{3}, 75 \mathrm{MHz}\right): \delta(\mathrm{ppm})=170.6,166.5$, 138.3, 132.2, 129.1, 128.7, 52.7, 41.9.

4.1.30. $N$-(p-Chlorobenzoyl)-glycinol $5 \boldsymbol{b}^{37}$ Following a literature procedure, ${ }^{18}$ ester 5c (488.2 mg, $\left.2.15 \mathrm{mmol}\right), \mathrm{NaBH}_{4}(408.8 \mathrm{mg}$, $10.75 \mathrm{mmol})$ and THF $(1.8 \mathrm{~mL})$ were stirred at $65{ }^{\circ} \mathrm{C}$ for 15 min. Then $\mathrm{MeOH}(1.2 \mathrm{~mL})$ was added and the resulting mixture was stirred for another $15 \mathrm{~min}$ at $65{ }^{\circ} \mathrm{C}$. The mixture was then cooled to room temperature, quenched with $\mathrm{HCl} 1 \mathrm{M}$ and extracted with $\mathrm{CH}_{2} \mathrm{Cl}_{2}$. The combined organic layers were dried over $\mathrm{MgSO}_{4}$, filtered and concentrated under reduced pressure to afford the expected alcohol $\mathbf{5 b}$ as a white solide in $89 \%$ yield ( $384.4 \mathrm{mg}$ ). The crude compound was found pure enough for further reactions. ${ }^{1} \mathrm{H}$ NMR $\left(\mathrm{CDCl}_{3}, 200 \mathrm{MHz}\right)$ : $\delta(\mathrm{ppm})=7.73-7.60(\mathrm{~m}, 2 \mathrm{H}), 7.39-7.27(\mathrm{~m}, 2 \mathrm{H}), 7.01(\mathrm{~s}, 1 \mathrm{H})$, $3.84-3.71(\mathrm{~m}, 2 \mathrm{H}), 3.55(\mathrm{dd}, J=10.2,5.2 \mathrm{~Hz}, 2 \mathrm{H}), 3.46(\mathrm{~s}, 1 \mathrm{H})$. ${ }^{13} \mathrm{C}$ NMR $\left(\mathrm{CDCl}_{3}, 75 \mathrm{MHz}\right): \delta(\mathrm{ppm})=167.7,138.1,132.6,128.9$, 128.6, 62.0, 42.9.

4.1.31. Moclobemide. ${ }^{38}$ Alcohol 5b (350 mg, $\left.1.97 \mathrm{mmol}\right)$, triethylamine ( $826 \mu \mathrm{L}, 5.93 \mathrm{mmol})$ DMAP $(24.1 \mathrm{mg}, 0.20 \mathrm{mmol})$, and sodium chloride (346.6 mg, $5.93 \mathrm{mmol}$ ) were stirred in $\mathrm{CH}_{2} \mathrm{Cl}_{2}$ $(9.9 \mathrm{~mL})$ and methanesulfonyl chloride $(460 \mu \mathrm{L}, 5.93 \mathrm{mmol})$ was added dropwise. The mixture was stirred overnight and completion of the reaction was assessed by HPLC. Reaction was quenched using a saturated solution of $\mathrm{NH}_{4} \mathrm{Cl}$ and extracted with $\mathrm{CH}_{2} \mathrm{Cl}_{2}$. The combined organic layers were washed with a saturated solution of $\mathrm{NaHCO}_{3}$, brine, dried over $\mathrm{MgSO}_{4}$ filtered and concentrated under reduced pressure. The crude chloride was filtered through a pad of silica using $\mathrm{CH}_{2} \mathrm{Cl}_{2}$ as eluant. Filtered compound (white solid, $450 \mathrm{mg}$ ) was found pure enough for further reaction. ${ }^{1} \mathrm{H}$ NMR $\left(\mathrm{CDCl}_{3}, 200 \mathrm{MHz}\right): \delta(\mathrm{ppm})=7.81-7.62(\mathrm{~m}, 2 \mathrm{H}), 7.47-7.33(\mathrm{~m}, 2 \mathrm{H})$, $6.69(\mathrm{~s}, 1 \mathrm{H}), 3.87-3.64(\mathrm{~m}, 4 \mathrm{H}) .{ }^{13} \mathrm{C} \mathrm{NMR}\left(\mathrm{CDCl}_{3}, 75 \mathrm{MHz}\right): \delta(\mathrm{ppm})=$ $166.7,138.2,132.6,129.1,128.6,44.2,41.9$. A mixture of compound 5a (109.0 mg, $0.5 \mathrm{mmol}$ ) and morpholine (348 $\mu \mathrm{L}, 4 \mathrm{mmol})$ was then stirred at $100{ }^{\circ} \mathrm{C}$ for $2 \mathrm{~h}$. After cooling to room temperature, $5 \mathrm{~mL}$ of $\mathrm{H}_{2} \mathrm{O}$ and then a $10 \%$ ammonia solution were added. The solution was extracted with $\mathrm{CH}_{2} \mathrm{Cl}_{2}$. The organic layers were combined, dried over $\mathrm{MgSO}_{4}$, filtered and evaporated under reduced pressure. Purification on silica gel using a mixture $\mathrm{CH}_{2} \mathrm{Cl}_{2} / \mathrm{MeOH}$ 95:5 afforded $56.6 \mathrm{mg}$ of moclobemide as a white solid (42\% yield). ${ }^{1} \mathrm{H} \mathrm{NMR}\left(\mathrm{CDCl}_{3}, 200 \mathrm{MHz}\right): \delta(\mathrm{ppm})=7.72(\mathrm{dd}, J=8.9,2.2 \mathrm{~Hz}, 2 \mathrm{H})$, 7.41 (dd, $J=8.9,2.2 \mathrm{~Hz}, 2 \mathrm{H}), 6.78(\mathrm{~s}, 1 \mathrm{H}), 3.78-3.69(\mathrm{~m}, 4 \mathrm{H}), 3.54$ (dd, $J=11.4,5.4 \mathrm{~Hz}, 2 \mathrm{H}), 2.67-2.56(\mathrm{~m}, 2 \mathrm{H}), 2.56-2.46(\mathrm{~m}, 4 \mathrm{H}) .{ }^{13} \mathrm{C} \mathrm{NMR}$ $\left(\mathrm{CDCl}_{3}, 75 \mathrm{MHz}\right): \delta(\mathrm{ppm})=166.4,137.6,133.0,128.8,128.4,66.9$, $56.9,53.4,36.2$.

\section{References and notes}

1. (a) Humphrey, J. M.; Chamberlin, A. R. Chem. Rev. 1997, 97, 2243-2266; (b) Carey, J. S.; Laffan, D.; Thomson, C.; Williams, M. T. Org. Biomol. Chem. 2006, 4 2337-2347.

2. (a) Allen, C. L.; Williams, J. M. J. Chem. Soc. Rev. 2011, 40, 3405-3415; (b) Dobereiner, G. E.; Crabtree, R. H. Chem. Rev. 2010, 110, 681-703; (c) Milstein, D. Top. Catal. 2010, 53, 915-923; (d) Chen, C.; Hong, S. H. Org. Biomol. Chem. 2011, 9, 20-26.

3. (a) Gunanathan, C.; Ben-David, Y.; Milstein, D. Science 2007, 317, 790-792; (b) Nordstrøm, L. U. Vogt, H.: Madsen, R. J. Am. Chem. Soc. 2008, 130 17672-17673; (c) Naota, T.; Murahashi, S.-I. Synlett 1991, 693-694; (d) Ghosh, S. C.; Muthaiah, S.; Zhang, Y.; Xu, X.; Hong, S. H. Adv. Synth. Catal. 2009, 351 2643-2649; (e) Zhang, J.; Senthilkumar, M.; Ghosh, S. C.; Hong, S. H. Angew. Chem., Int. Ed. 2010, 49, 6391-6395; (f) Zeng, H.; Guan, Z. J. Am. Chem. Soc 2011, 133, 1159-1161.

4. (a) Fujita, K.-i.; Takahashi, Y.; Owaki, M.; Yamamoto, K.; Yamaguchi, R. Org. Lett. 2004, 6, 2785-2788; (b) Zweifel, T.; Naubron, J. V.; Grutzmacher, H. Angew. Chem., Int. Ed. 2009, 48, 559-563.

5. Soulé, J.-F.; Miyamura, H.; Kobayashi, S. J. Am. Chem. Soc. 2011, 133 $18550-18553$

6. Klitgaard, S. K.; Egeblad, K.; Mentzel, U. V.; Popov, A. G.; Jensen, T.; Taarning, E.; Nielsen, I. S.; Christensen, C. H. Green. Chem. 2008, 10, 419-423.

7. Wang, Y.; Zhu, D.; Tang, L.; Wang, S.; Wang, Z. Angew. Chem., Int. Ed. 2011, 50, 8917-8921.

8. Shimizu, K.-i.; Ohshima, K.; Satsuma, A. Chem.-Eur. J. 2009, 15, 9977-9980.

9. Anastas, P. T.; Warner, J. C. Green Chemistry: Theory and Practice; Oxford University: Oxford, UK, 1998.

10. (a) Yoo, W. J.; Li, C. J. J. Am. Chem. Soc. 2006, 128, 13064-13065; (b) Reddy, K. R.; Maheswari, C. U.; Venkateshwar, M.; Kantam, M. L. Eur. J. Org. Chem. 2008, 3619-3622; (c) Tank, R.; Pathak, U.; Vimal, M.; Bhattacharyya, S. Pandey, L. K. Green. Chem. 2011, 13, 3350-3354; (d) Ghosh, S. C.; Ngiam, J. S. Y.; Chai, C. L. L.; Seayad, A. M.; Dang, T. T.; Chen, A. Adv. Synth. Catal. 2012, 354, 1407-1412; (e) Ghosh, S. C.; Ngiam, J. S. Y.; Seayad, A. M.; Tuan, D. T.; Chai, C. L. L.; Chen, A. J. Org. Chem. 2012, 77, 8007-8015; (f) Cadoni, R.; Porcheddu, A.; Giacomelli, G.; De Luca, L. Org. Lett. 2012, 14 5014-5017; (g) Porcheddu, A.; De Luca, L. Adv. Synth. Catal. 2012, 354 2949-2953.

11. (a) Bonnamour, J: Métro, T.-X.: Martinez, J: Lamaty, F. Green Chem 2013, 15 1116-1120; (b) Métro, T.-X.; Bonnamour, J.; Reidon, T.; Sarpoulet, J.; Martinez, J.; Lamaty, F. Chem. Commun. 2012, 11781-11783; (c) Petiot, P.; Charnay, C.; Martinez, J.; Puttergill, L.; Galindo, F.; Lamaty, F.; Colacino, E. Chem. Commun. 2010, 46, 8842-8844; (d) Declerck, V.; Nun, P.; Martinez, J.; Lamaty, F. Angew. Chem. Int. Ed. 2009, 48, 9318-9321.

12. Bantreil, X.; Fleith, C.; Martinez, J.; Lamaty, F. ChemCatChem 2012, 4, $1922-1925$.

13. Wu, X.-F.; Sharif, M.; Pews-Davtyan, A.; Langer, P.; Ayub, K.; Beller, M. Eur. J. Org. Chem. 2013, 2783-2787.

14. Gaspa, S.; Porcheddu, A.; De Luca, L. Org. Biomol. Chem. 2013, 11, 3803-3807.

15. Ghosh, S. C.; Ngiam, J. S. Y.; Seayad, A. M.; Tuan, D. T.; Johannes, C. W.; Chen, A Tetrahedron Lett. 2013, 54, 4922-4925.

16. Wu, X.-F.; Bheeter, C. B.; Neumann, H.; Dixneuf, P. H.; Beller, M. Chem. Commun 2012, 12237-12239.

17. Arnold, K.; Davies, B.; Giles, R. L.; Grosjean, C.; Smith, G. E.; Whiting, A. Adv Synth. Catal. 2006, 348, 813-820.

18. Gonçalves, R. S. B.; Pinheiro, A. C.; Silva, E. T. D.; Costa, J. C. S. D.; Kaiser, C. R.; Souza, M. V. N. D. Synth. Commun. 2011, 41, 1276-1281.

19. Burkard, W.; Wyss, P.-C. US4210754 (A).

20. Kitamura, M.: Suga, T.; Chiba, S.; Narasaka, K. Org Lett 2004, 6, 4619-4621.

21. Zhang, M.; Vedantham, P.; Flynn, D. L.; Hanson, P. R. J. Org. Chem. 2004, 69 8340-8344.

22. Sharma, S.; Park, E.; Park, J.; Kim, I. S. Org. Lett. 2012, 14, 906-909.

23. Shangguan, N.; Katukojvala, S.; Greenberg, R.; Williams, L. J. J. Am. Chem. Soc 2003, 125, 7754-7755.

24. De, C. K.; Klauber, E. G.; Seidel, D. J. Am. Chem. Soc. 2009, 131, 17060-17061.

25. Lee, K.-Y.; Kim, Y.-H.; Park, M.-S.; Oh, C.-Y.; Ham, W.-H. J. Org. Chem. 1999, 64 9450-9458.

26. Niwa, T.; Suehiro, T.; Yorimitsu, H.; Oshima, K. Tetrahedron 2009, 65 $5125-5131$

27. Kita, Y.; Akai, S.; Ajimura, N.; Yoshigi, M.; Tsugoshi, T.; Yasuda, H.; Tamura, Y. J. Org. Chem. 1986, 51, 4150-4158.

28. a) Jeh-Jian, H.; Hung-Cheh, C. Phytochemistry 1995, 39, 899-902; b) Minor, D L.; Wyrick, S. D.; Charifson, P. S.; Watts, V. J.; Nichols, D. E.; Mailman, R. B. J. Med. Chem. 1994, 37, 4317-4328.

29. Park, H.-G.; Kim, M.-J.; Park, M.-K.; Jung, H.-J.; Lee, J.; Choi, S.-H.; Lee, Y.-J.; Jeong, B.-S.; Lee, J.-H.; Yoo, M.-S.; Ku, J.-M.; Jew, S.-S. J. Org. Chem. 2005, 70 1904-1906.

30. Maezaki, N.; Furusawa, A.; Uchida, S.; Tanaka, T. Tetrahedron 2001, 57, 9309-9315.

31. Cazorla, C.; Métay, E.; Lemaire, M. Tetrahedron 2011, 67, 8615-8621.

32. Kasashima, Y.; Uzawa, A.; Hashimoto, K.; Yokoyama, Y.; Mino, T.; Sakamoto, M.; Fujita, T. J. Oleo Sci. 2010, 59, 607-613.

33. Wang, Z.; Zhang, Y.; Fu, H.; Jiang, Y.; Zhao, Y. Org. Lett. 2008, 10, 1863-1866.

34. Yokomatsu, T.; Arakawa, A.; Shibuya, S. J. Org. Chem. 1994, 59, 3506-3508. 
35. Arias, H. R.; Gu, R.-X.; Feuerbach, D.; Guo, B.-B.; Ye, Y.; Wei, D.-Q. Biochemistry 2011, 50, 5263-5278.

36. Hanzawa, Y.; Kasashima, Y.; Tomono, K.; Mino, T.; Sakamoto, M.; Fujita, T. J. Oleo Sci. 2012, 61, 393-399.
37. Ohshima, T.; Hayashi, Y.; Agura, K.; Fujii, Y.; Yoshiyama, A.; Mashima, K. Chem. Commun. 2012, 5434-5436.

38. Kim, D.; Sambasivan, S.; Nam, H.; Hean Kim, K.; Yong Kim, J.; Joo, T.; Lee, K.-H.; Kim, K.-T.; Han Ahn, K. Chem. Commun. 2012, 6833-6835. 